\title{
Alveolar bone growth with orthoeruption
}

\begin{abstract}
This teenage patient presents with pronounced esthetic and functional dental concerns. An anterior frontal view of the patient's dentition shows virtually total facial blockage of the maxillary right central incisor creating mesial contact of the right lateral incisor with the left central incisor immediately lingual to the blocked out right central incisor. A similar severe misalignment takes place with the orientation of the mandibular left canine and adjacent mandibular left premolar and mandibular left lateral incisor. In addition, the patient exhibits a pronounced overbite with maxillary anterior teeth entirely covering the opposing mandibular teeth and with an overjet of $4 \mathrm{~mm}$. This case report illustrates the potential to grow alveolar bone with the orthodontic systems known as Fastbraces ${ }^{\circledR}$ Technologies that is based on the non-extraction mechanically aided continuation of eruption by moving the roots towards their final position from the onset of therapy. Post treatment clinical photos and radiographic results show dramatic esthetic and functional improvement with the elimination of the severe pretreatment crowding and occlusal discrepancy with the establishment of a stable occlusion and good intercuspation.
\end{abstract}

Volume 7 Issue 5 - 2017

\author{
Anthony D Viazis,' Evangelos Viazis, ${ }^{2}$ Tom C \\ Pagonis $^{3}$ \\ 'Orthodontist, Private Practice, USA \\ ${ }^{2}$ Private Practice, Greece \\ ${ }^{3}$ Department of Restorative Dentistry and Biomaterials \\ Sciences, Harvard School of Dental Medicine, USA
}

\begin{abstract}
Correspondence: Tom C Pagonis, Department of Restorative Dentistry and Biomaterials Sciences, Harvard School of Dental Medicine, 188 Longwood Avenue, Boston, MA 02115, USA, Tel 1617-432-5846, Fax 1617-432-0901, Email dental.research@comcast.net
\end{abstract}

Received: July 14, 2017 | Published: July 25, 2017

Keywords: severe crowding, orthoeruption, orthodontics, braces, alveolar bone growth

\section{Introduction}

While classifying the degree of severe anterior crowding is subjective, the dichotomy of orthodontic treatment planning with or without extractions dates back to the 1890's when Angle, who introduced classifications of malocclusions, initially advocated nonextraction orthodontic therapy. ${ }^{1}$ Subsequently, Calvin Case challenged Angle's assertion that the presence of all teeth was necessary to produce balance and harmony and argued that mechanical forces could not induce basal bone growth beyond the developed or inherent size. ${ }^{2}$ In 1944, Angle's student, Charles Tweed noted a high prevalence of relapse in his cases and abandoned his mentor's teachings of nonextraction therapy. ${ }^{3}$ The dichotomy of extraction vs non-extraction in orthodontic treatment planning remains today even when considering diagnostic elements such as molar relationship, tooth-arch discrepancy or cephalometric discrepancy and facial profile. This case report illustrates the potential to grow alveolar bone with the orthodontic
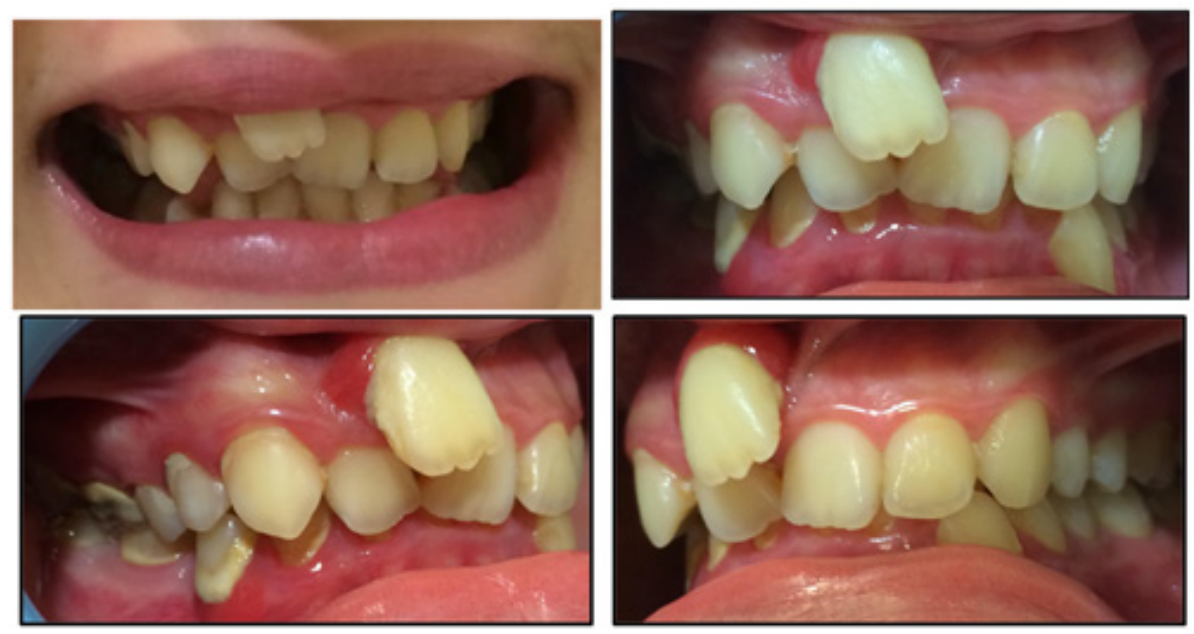

Figure I (A) Pre-treatment intra-oral frontal view photographs.

systems known as Fastbraces ${ }^{\circledR}$ Technologies that is based on the nonextraction mechanically aided continuation of eruption by moving the roots towards their final position from the onset of therapy.

\section{Case report}

\section{Diagnosis}

This young teenage patient presented to the second author's private practice in Athens, Greece with a chief complaint of esthetic concerns and with difficulty chewing (Figure 1). On examination the patient has a mesoproscopic facial form with an overbite of $5 \mathrm{~mm}$ and an overjet of $4 \mathrm{~mm}$. Of note, the patient's maxillary anterior dentition shows virtually total facial blockage of the maxillary right central incisor creating mesial contact of the right lateral incisor with the left central incisor immediately lingual to the blocked out right central incisor. The mandibular arch also exhibits severe crowding in the area of the mandibular left canine. 

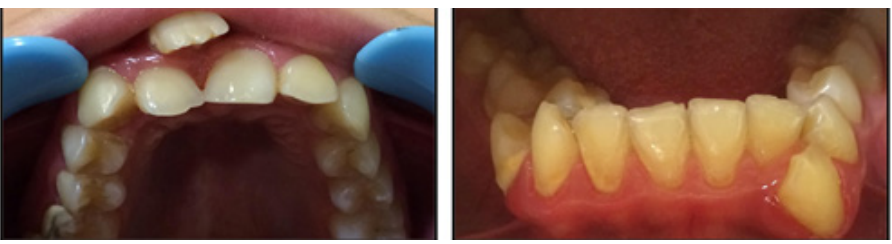

Figure I (B) Pre-treatment intra-oral occlusal view photographs.

\section{Treatment objectives}

Upon completion of the clinical examination and review of the pre-treatment panoramic and lateral cephalogram radiographs (Figure 2), a non-extraction treatment regimen was recommended due to the favorable prognosis of alveolar bone growth with a new form of orthodontic tooth movement that involves the immediate activation of the roots toward their final naturally erupted positions. This treatment was recommended in order to correct the severe crowding, obtain proper overjet and overbite relations, level and align the occlusion and restore satisfactory esthetics by utilizing the bracket systems of Fastbraces ${ }^{\circledR}$ Technologies.


Figure 2 Pre-treatment lateral cephalogram and panoramic radiographs.

\section{Treatment progress}

At the initial treatment appointment, four brackets were placed on the maxillary anterior teeth for patient comfort and the patient was followed every 21 days for a period of four months (Figure 3). This initial set up along with the subsequent addition of full maxillary and mandibular brackets (Figure 4) utilizing the orthodontic systems of Fastbraces ${ }^{\circledR}$ Technologies is designed to provide appropriate force by inducing alveolar bone growth to not only accommodate the severely misaligned maxillary right central incisor but accommodate all teeth in their respective arches. Total treatment time with full orthodontic brackets took a little over 12 months with appointments scheduled approximately on a monthly basis with minor interproximal reduction mesial to all canine teeth.
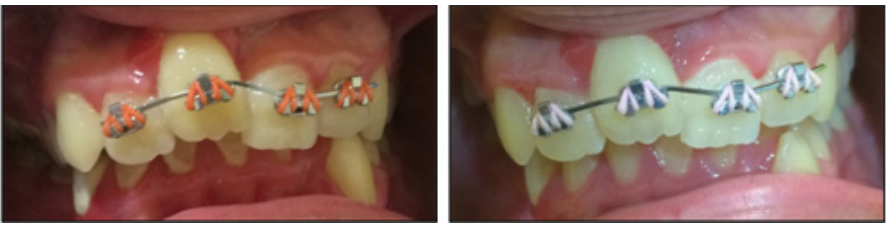

Figure 3 Treatment progress with four maxillary anterior brackets.

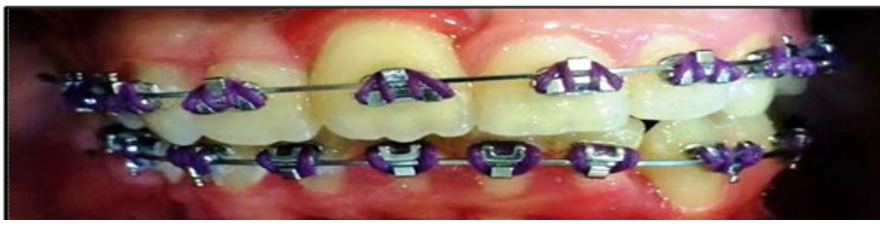

Figure 4 Treatment progress with full maxillary and mandibular brackets.
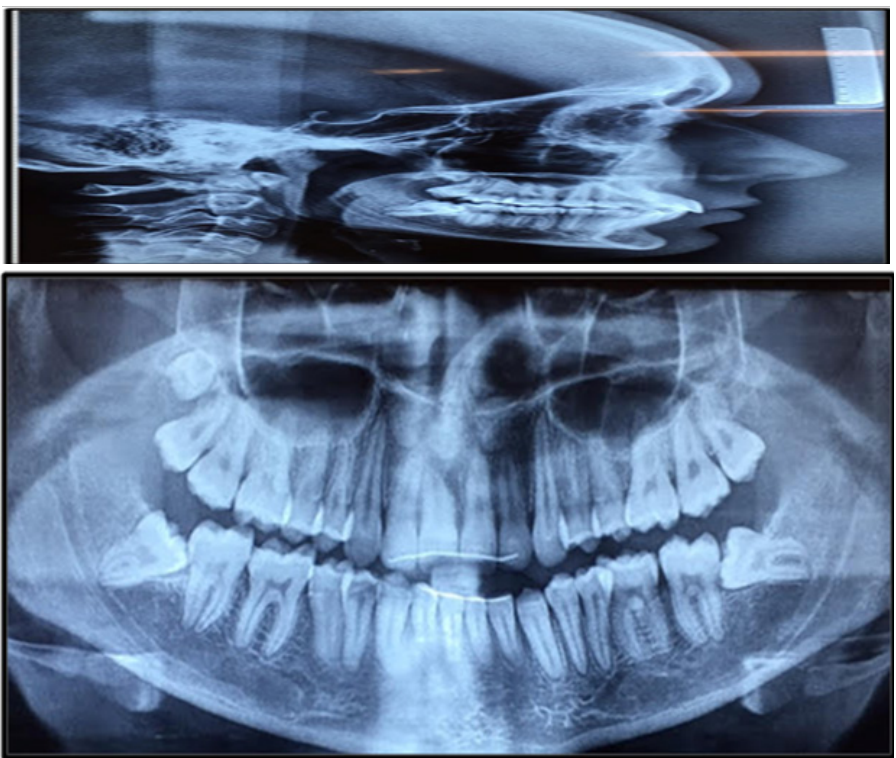

Figure 5 Post treatment lateral cephalogram and panoramic radiographs.
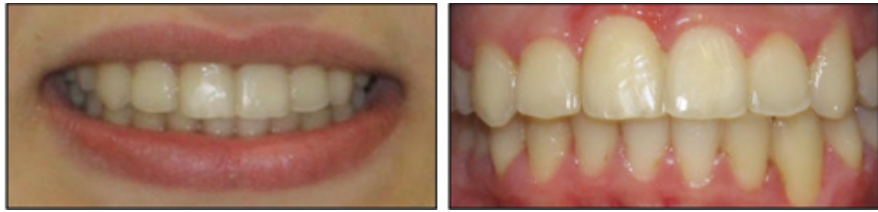

Figure 6 Post treatment facial and intra-oral frontal view photographs.
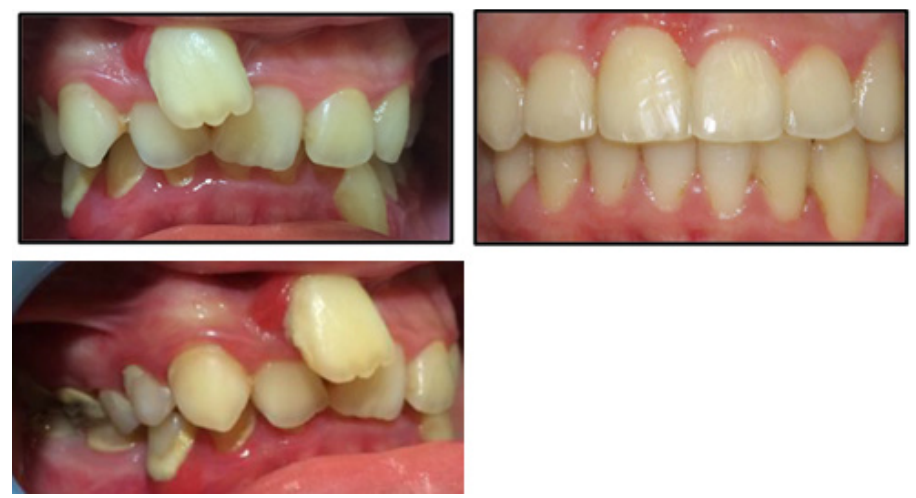


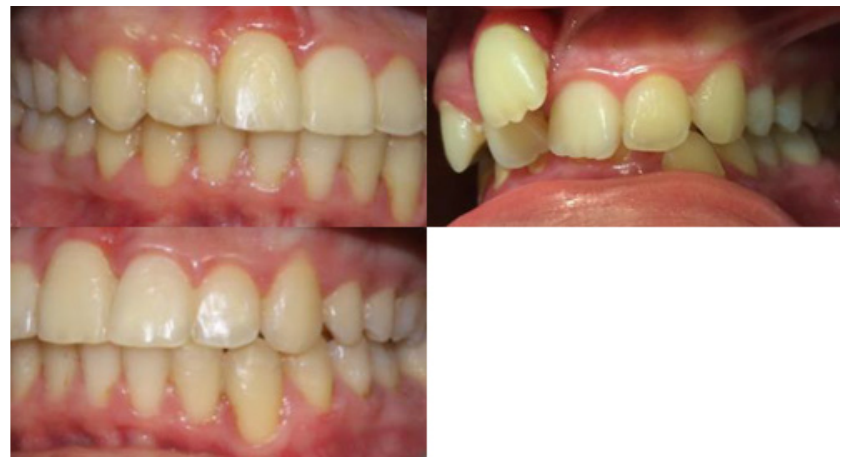

Figure 7 (A) Comparing Before and After frontal, right and left buccal photographs.

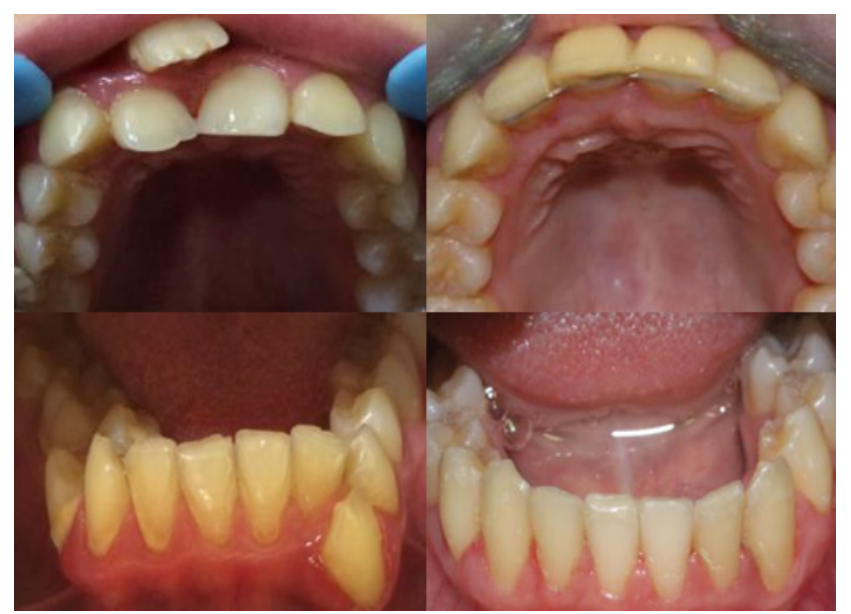

Figure 7 (B) Comparing Before and After maxillary and mandibular occlusal photographs.

\section{Treatment results}

Clinical results along with post treatment radiograph (Figure 5) and photographs (Figures 6\&7) show dramatic_esthetic improvement, particularly with alveolar bone development which allowed the alignment of the right maxillary central incisor into its natural position. Post treatment results also show a stable occlusion with proper over jet and over bite relations.

\section{Discussion}

In 2014, Viazis et al., ${ }^{4}$ introduced the biologically based orthodontic diagnostic terms of Orthodontosis and Orthodontitis. ${ }^{4}$ Orthodontosis is defined as the non-inflammatory deficiency of alveolar bone in the horizontal dimension caused by the displaced $\operatorname{root}(\mathrm{s})$ of the tooth, typically palatally or lingually. Orthodontitis is defined as associated excess soft tissue manifestation and chronic inflammation. In effect the hard tissue bony hypoplasia (Orthodontosis) and soft tissue manifestation (Orthodontitis) associated with malpositioned roots represent unfinished eruption. Based upon these definitions, orthodontic treatment should be directed towards mimicking and continuing the light forces of natural eruption possibly stimulating bone remodeling around displaced roots thereby eliminating the need for extraction therapy. Furthermore, this mechanically assisted continuation of eruption has been defined as "orthoeruption" in the literature ${ }^{4}$ and allows for the up-righting of displaced roots into a straight position as if the teeth erupted in that position. Therefore, orthoeruption results in the alveolar bone remodeling and restoration of the dental arch to its appropriate natural size and shape. Accordingly, non-extraction therapy is almost always achieved through this alveolar bone growth as the alveolar bone reacts to a tooth erupting in its correct place in the arch.

The clinical pre-treatment presentation of this case especially with regard to the maxillary right central incisor clearly shows the lack and perhaps more accurately, the absence of alveolar bone on the proximal and facial sides. Even today and with this clinical presentation, the century plus old dichotomy of extraction vs nonextraction in orthodontic treatment would lead most orthodontic clinicians to extraction therapy based upon the out dated concept forwarded by Calvin Case which maintained that alveolar bone has little or no capacity to grow with traditional orthodontic mechanical forces. This case report along with other studies in the literature ${ }^{5-8}$ illustrates the potential to grow alveolar bone with the orthodontic systems of Fastbraces ${ }^{\circledR}$ Technologies which is based on the nonextraction mechanically aided continuation of eruption by moving the roots toward their final position from the onset of therapy.

By definition natural eruption is root movement which is followed by alveolar bone growth. The new bone around the final position of the naturally erupted root demonstrates the alveolar bone growth that occurred during eruption. If not, the root would find itself outside the alveolar bone housing. So a defacto consequence of root movement in natural eruption is the alveolar bone growth around the new position. Much like the remodeling of alveolar bone to accommodate teeth during natural eruption, orthodontically induced eruption or orthoeruption may stimulate the continued remodeling of alveolar bone to accommodate the roots towards their final naturally erupted position.

\section{Conclusion}

This case report demonstrates the successful non-extraction orthodontic correction, of severe maxillary anterior crowding or localized orthodontosis and shows the dramatic esthetic and functional improvement (Figure 7) with the establishment of a stable occlusion and good intercuspation with Fastbraces ${ }^{\circledR}$ Technologies. These new technology systems of braces facilitate the continuation of eruption while inducing alveolar bone remodeling and development in short treatment times by moving the tooth roots toward their final naturally erupted position from the beginning of treatment. This orthodontically induced eruption of teeth results in the successful completion of cases non-extraction.

\section{Acknowledgments}

None.

\section{Conflicts of interest}

The author declares that there is no conflict of interest.

\section{References}

1. Angle EH. Classification of malocclusion. Dental Cosmos. 1899;41:248226.

2. Bernsteim L. Edward H. Angle versus Calvin S. Case: extraction versus nonextraction. Historical revisionism. Part II. Am J Orthod Dentofacial Orthop. 1992;102(6):546-551. 
3. Tweed C. Indications for extraction of teeth in orthodontic procedure. Am J Orthod Dentofacial Orthop. 1944;30(8):405-428.

4. Viazis AD, Viazis E, Pagonis TC. The Concept of a New Dental Disease: Orthodontosis and Orthodontitis. J Dent Health Oral Disord Ther. 2014;1(5):00030

5. Viazis AD, Viazis E, Pagonis TC. The Fallacy of Tongue Thrust and NonSurgical Treatment of a Severe Anterior Open Bite. J Dent Health Oral Disord Ther. 2016;4(4):0120.
6. Viazis AD, Viazis E, Pagonis TC. Non-Surgical Orthodontic Treatment of an Orthognathic Surgical Case. J Dent Health Oral Disord Ther 2016;4(5):00128

7. Viazis AD, Viazis E, Pagonis TC. Non-surgical Orthodontic Adult Molar Crossbite Correction and Sleep Apnea. J Dent Health Oral Disord Ther. 2016;5(5):00168.

8. Viazis AD, Thaveeprungsiporn M, Pagonis TC. Alveolar Bone Remodeling and Development after Immediate Orthodontic Root Movement. J Dent Health Oral Disord Ther. 2017;(2):00195. 